\title{
Inactivation of Hydrogen Peroxide by Vitamin A, Vitamin C, Vitamin E, and Other Commercial Items Through Pe- roxidase-Catalyzed Reaction
}

\author{
Makayla Gomperts ${ }^{1}$ \\ ${ }^{1}$ Perry Meridian High School, Indianapolis, Indiana, USA
}

\begin{abstract}
Reactive oxygen species (ROS), such as hydrogen peroxide $\left(\mathrm{H}_{2} \mathrm{O}_{2}\right)$, are highly reactive and can inhibit the normal function of cells. Due to the damage ROS can cause, much interest has been generated around the effects of antioxidant supplements, which could potentially prevent or slow down ROS in the body. In this study, a variety of antioxidants were evaluated for their ability to inactivate $\mathrm{H}_{2} \mathrm{O}_{2}$ in a peroxidase-catalyzed reaction. Six of the fourteen antioxidants were significantly effective at inactivating $\mathrm{H}_{2} \mathrm{O}_{2}$ compared to the control. This study should be used to further understand how antioxidant supplements could be beneficial in the human diet.
\end{abstract}

\section{Introduction}

The Free Radical Theory, as discovered by Denham Harman, explains that when there is a presence of reactive oxygen species (ROS) and free radicals in the human body, it can cause progressive and irreversible oxidative damage. ${ }^{1,2}$ This damage has been suggested to be a cause of many human diseases such as cardiovascular disease, neurodegenerative disorders, stroke, diabetes, cancer, and alterations in DNA and genetic makeup. ${ }^{3}$ Additionally, many studies have suggested that ROS may even play a role in the aging process. ${ }^{3,4}$

ROS are chemical species that contain oxygen and are highly reactive, such as $\mathrm{H}_{2} \mathrm{O}_{2}$ (hydrogen peroxide). Free radicals, a molecular species derived from ROS, contain an unpaired electron, making the molecule highly reactive. ${ }^{5,6}$ ROS and free radicals are known to be very harmful to the human body, as they induce a state known as oxidative stress. ${ }^{3}$ Oxidative stress is a physiological change in biological properties that can inhibit normal cell function and increase the damage to tissues and muscle. ${ }^{6}$ When the human body is subject to ROS, much damage can be made to negatively impact cell membranes, lipids, proteins, and DNA. ${ }^{3,5,7}$

To combat this damage, the body has developed an antioxidant defense system in an attempt to prevent the formation of ROS. ${ }^{3,7,8,9}$ An antioxidant is a substance which slows or prevents the oxidation of another substance, therefore, reducing the amount of ROS. Enzymatic defenses are the body's evolved defense systems against ROS; this includes enzymes formed like peroxidase, which is meant to break down $\mathrm{H}_{2} \mathrm{O}_{2}$, the toxic byproduct created when oxygen is used in a chemical reaction. ${ }^{5,7} \mathrm{H}_{2} \mathrm{O}_{2}$ will be the focus of this research because it is an easily accessible form of a ROS. Enzymes help catalyze and speed up chemical reactions without being used up in the process. Additionally, there is a non-enzymatic defense system in which ingested vitamins from foods and other dietary sources can protect damage from ROS. ${ }^{5,7}$ Juices, teas, and wines are known to be a crucial source of antioxidants for humans because they contain many vitamins. This has generated much interest and research in determining whether supplements could be used to potentially prevent further damage from ROS and other human diseases. ${ }^{5}$ 


\section{Literature Review}

Michael King, a professor of medical education at the Indiana University School of Medicine, found that Vitamin C, Vitamin A, and Vitamin E all have antioxidant properties that can significantly aid in the reduction of reactive oxygen species, especially $\mathrm{H}_{2} \mathrm{O}_{2}$. He additionally notes how each vitamin can be found in a wide variety of forms. For example, Vitamin $\mathrm{C}$ is available both directly through a tablet or capsule or indirectly through a collection of fruits and vegetables. ${ }^{10}$ Members from the Department of Food Technology and Science at the Federal University of Santa Maria contribute to this idea by explaining that fruits and vegetables contain a type of compound called polyphenols. ${ }^{11} \mathrm{~A}$ polyphenol can be defined as a natural antioxidant that can help to slow or stop the oxidative damage caused by ROS. ${ }^{12}$ Discussion centered around these fruits has increased because of their antioxidant properties.

In a study comparing many fruits and their response to different free radicals, Shiow Wang, a research plant physiologist, found that blackberries had the highest inhibition of $\mathrm{H}_{2} \mathrm{O}_{2}$ activity in comparison to cranberries which had the lowest. ${ }^{13}$ Maria Gil, who holds a PhD in biology, added on to this research by revealing that commercial pomegranate juice, which can be found in grocery stores, has an antioxidant activity three times higher than wine and green tea. ${ }^{14}$ Many additional studies have attempted to test both juice and the seeds of fruits in response to $\mathrm{H}_{2} \mathrm{O}_{2}$ through in vivo assays, although scientists have been met with extremely equivocal results. ${ }^{11,12,13,14,15,16,17,18,19,20,21,22}$

In a study titled, "Role of ROS and Nutritional Antioxidants in Human Diseases," Zewen Liu, a member of the department of anesthesiology at Wuhan University, explains:

"Antioxidant supplementation has been shown to attenuate endogenous antioxidant depletion, thus alleviating associated oxidative damage in some clinical research. However, some results indicate that antioxidants exert no favorable effects on disease control."1

Liu's study and conclusions point to the idea that antioxidants may have few to none beneficial qualities or effects. This is supported in Goran Bjelakovic's research, as he and a team found that in 47 low bias trials, the given antioxidant treatments significantly increased mortality. ${ }^{23}$ Bjelakovic holds a M.D. degree and teaches at the University of Niš. This differs from studies conducted by both Sibel Konyalioglu, a biochemistry professor, and Alessandra Bosutti, a research associate at Manchester Metropolitan University, who discovered that resveratrol, an antioxidant commonly found in high concentrations in red grapes, can reduce oxidative stress caused by $\mathrm{H}_{2} \mathrm{O}_{2}{ }^{19,20}$

The National Institutes of Health attributes the contradicting nature of literature in the field to the fact that few high-quality clinical trials and in vivo assays have been conducted to date. ${ }^{24,25}$ Serge Hercberg, a professor of nutrition, supports this idea by claiming that even trials that include a placebo control have not provided clear evidence of a beneficial effect of supplements that contain antioxidants. ${ }^{26}$

Ian Young, a member of the clinical biochemistry department at the Institute of Clinical Science, addresses this issue by explaining there is a lot of evidence that explains the process of oxidative stress and the potential role that an antioxidant defense system has in protecting against this damage, but he suggests the need for a more comprehensive "understanding of the biochemical events occurring at a cellular level to influence oxidative damage" in order "to guide future therapeutic advances." ${ }^{27}$ Because I am not able to perform research on living organisms, my study will instead be performed in vitro in order to provide a more complete overview of the chemistry behind the ROS, $\mathrm{H}_{2} \mathrm{O}_{2}$, and how antioxidants can play a role in mitigating its harmful effects. Additionally, there are few studies that compare both antioxidants in a vitamin or tablet form as well as a juice form, so I will attempt to fill in this gap by comparing a wide variety of products that contain antioxidant properties and how they influence the damage caused by $\mathrm{H}_{2} \mathrm{O}_{2}$.

The goal is to conduct a study that answers the following question: How do antioxidants such as Vitamin A, Vitamin C, Vitamin E, and resveratrol comparatively help in inactivating hydrogen peroxide and limiting its negative effects? 


\section{Methodology}

The majority of this study fell under the realm of chemistry with a small amount of both biology and nutrition. An experimental quantitative research study was conducted through the analysis of the byproducts of a peroxidase-catalyzed reaction when introduced to different amounts of several forms of various antioxidants. This research method enabled the ability to analyze the data being collected and measurements being observed with great detail. Refer to Appendix A for more definitions.

The primary focus was to collect quantitative data in order to analyze and compare the results with each other to determine which antioxidant and in which form was the most helpful in mitigating the damage of $\mathrm{H}_{2} \mathrm{O}_{2}$. The research was performed in vitro as opposed to in vivo due to a lack of time and resources; however, this assay will help to provide a more comprehensive understanding of the chemistry behind this topic. Rather than conducting research on a human or animal subject, the use of a chemical reaction was the main means of examining the effects of the antioxidants on mitigating the damage of ROS.

For the purposes of this experiment, the following peroxidase-catalyzed chemical reaction was focused on.

$$
2 \mathrm{H}_{2} \mathrm{O}_{2}+2 \mathrm{AH}_{2} \rightarrow 4 \mathrm{H}_{2} \mathrm{O}+\mathrm{A}_{2}
$$

Table 1. Explanation of Peroxidase-catalyzed Reaction

\begin{tabular}{|l|l|l|}
\hline Molecule & Name & Definition and Function \\
\hline $\mathrm{A}_{2}$ & Oxidized Substrate & $\begin{array}{l}\text { Substance that was tested and measured over time, will gain electrons } \\
\text { from reducing agents }\end{array}$ \\
\hline $\mathrm{AH}_{2}$ & Reducing Agent & $\begin{array}{l}\text { Substance that transferred hydrogen atoms and electrons to hydrogen } \\
\text { peroxide }\end{array}$ \\
\hline $\mathrm{H}_{2} \mathrm{O}$ & Water & Byproduct of the chemical reaction \\
\hline $\mathrm{H}_{2} \mathrm{O}_{2}$ & Hydrogen Peroxide & ROS that is highly reactive, toxic, and harmful \\
\hline
\end{tabular}

In this study, $\mathrm{AH}_{2}$ represents guaiacol, a substance that donated hydrogen to the $\mathrm{H}_{2} \mathrm{O}_{2}$, thus becoming oxidized. ${ }^{28,29} \mathrm{~A}_{2}$ represents tetraguaiacol, a brown product, which was produced when oxidized guaiacol molecules bonded to each other. ${ }^{28,29}$ In the natural reaction that occurs in the body, the created product is clear and therefore unmeasurable. For this reason, guaiacol was added to change the product from a clear to brown color, making it tangible to measure. The substrate did not change any properties of the reaction, but instead made the rate of the reaction possible to be tested. This equation was considered the control because there was no antioxidant present. In this study, with the addition of other reagents such as the antioxidants, the previous reaction can be more accurately defined as the following.

$$
\left[\mathrm{H}_{2} \mathrm{O}_{2}+\mathrm{AA}\right]+\text { Guaiacol } \rightarrow \mathrm{H}_{2} \mathrm{O}+\text { Oxidized Guaiacol }
$$

AA represents the antioxidant. The $\mathrm{H}_{2} \mathrm{O}_{2}$ and antioxidant were incubated together for a set period of time. Several antioxidants were added, to test if they reduced the oxidized guaiacol produced over time. $\mathrm{The}^{\mathrm{H}} \mathrm{H}_{2} \mathrm{O}_{2}$ amount used was calculated to make it a limiting reactant, so that it had no effect on the reaction. Then, after the set incubation time, the guaiacol was added. For a chemical reaction to occur, an enzyme needed to be added to catalyze the reaction. For the purposes of this study, peroxidase was added, an enzyme in the body that naturally helps to break down $\mathrm{H}_{2} \mathrm{O}_{2}{ }^{30}$ The primary focus was measuring the amount of oxidized product, or guaiacol, that was produced over time during the reaction. The less accumulation and the smaller the slope of the reaction, the more beneficial the antioxidants were in the protection against $\mathrm{H}_{2} \mathrm{O}_{2}{ }^{28}$ 
Three different trials were performed with each form of each antioxidant to look at the average accumulation of guaiacol left in a cuvette, as $\mathrm{H}_{2} \mathrm{O}_{2}$ was mixed with several different reagents. Multiple trials were conducted in order to ensure that an adequate amount of data was gathered to determine a tested and verified conclusion; however, due to limited time and resources, only three trials were conducted for each. A SpectrovisPlus spectrophotometer was used at $25^{\circ} \mathrm{C}$ and $470 \mathrm{~nm}$ in conjunction with LoggerPro Software to determine the slope of each reaction for later statistical use. The following protocol and methodology that was used throughout this research was adapted from previous assays to fit the purpose of this experiment. ${ }^{28,29,30}$ The experiment was performed at Franklin College, using supplies and technology provided by the institute, under the direction of Dr. Larry Mordan.

\section{Ethics and Consent}

Because this study was experimental and required the use of chemicals, ethical concerns were reviewed. Approval from the IRB was received on 10/1/19. The consent showed I was ethically able to begin the experimentation and research that I performed.

\section{Antioxidants}

The antioxidants that were tested include Vitamin A, Vitamin C, Vitamin E, and resveratrol. They are commonly found in everyday commercial items such as juices and wines, so for the purpose of accessibility, some of these forms were used during testing. For this study, these items were referred to as antioxidants throughout the rest of this paper. Commercial items were tested as $100 \%$ solutions; however, the vitamins were not. The concentrations of Vitamin C, Vitamin A (2), and Vitamin E (2) are representative of the concentrations of these vitamins that appear in the blood stream. The abbreviations used in Table 2 will be used in all information that follows.

Table 2. List of Antioxidants and Commercial Items Used

\begin{tabular}{|l|l|}
\hline Abbreviation & Name and Form of Item Being Tested \\
\hline Vitamin A (1) & $1 \%$ solution \\
\hline Vitamin E (1) & $1 \%$ solution \\
\hline Vitamin C & $5 \mathrm{mg} / \mathrm{mL}$ concentration \\
\hline Vitamin A (2) & $0.4 \mu \mathrm{g} / \mathrm{mL}$ concentration \\
\hline Vitamin E (2) & $10 \mu \mathrm{g} / \mathrm{mL}$ concentration \\
\hline White Wine & Oliver Vineyard's Soft White Wine* \\
\hline Red Wine & Oliver Vineyard's Soft Red Wine* \\
\hline Rose Wine & Wedded Bliss (Next Chapter Winery in New Prague, MN)* \\
\hline Cranberry (RW) & R.W. Knudsen Cranberry Juice* \\
\hline Pomegranate (RW) & R.W. Knudsen Pomegranate Juice* \\
\hline Concord (RW) & R.W. Knudsen Organic Concord Grape Juice* \\
\hline Bai Pom & Bai Antioxidant Infusion Ipanema Pomegranate Juice* \\
\hline Bai Tea & Bai Antioxidant Infusion Socorro Tea* \\
\hline Cranberry Kroger & Kroger Brand Cranberry Juice Cocktail* \\
\hline
\end{tabular}

* Items were used as 100\% solutions.

\section{Preparing the Reagents}

To prepare the guaiacol, $50 \mathrm{~mL}$ of water and $110 \mu \mathrm{l}$ of guaiacol were mixed in a $45 \mathrm{~mL}$ centrifuge tube. Then, the components were shaken to mix well for approximately one minute. To prepare the $\mathrm{H}_{2} \mathrm{O}_{2}, 15 \mathrm{~mL}$ of a $3 \% \mathrm{H}_{2} \mathrm{O}_{2}$ solution and $30 \mathrm{~mL}$ of water were added in a $45 \mathrm{~mL}$ centrifuge tube. The components were shaken to mix well for approximately one minute. When preparing the peroxidase, $20 \mathrm{~g}$ out of the outer peeled portion of a turnip was cut and put in 
a blender. $300 \mathrm{~mL}$ of water was added, and the mixture was thoroughly blended for about one minute. The solution was filtered through multiple layers of cheesecloth into a conical flask in order to remove the remaining large sections of turnip. Additionally, a buffer solution was used in order to maintain the optimal $\mathrm{pH}$ for the reaction in order that it functioned properly. A $6.0 \mathrm{pH}$ buffer solution was used, as this is near the optimal $\mathrm{pH}$ for the enzyme to function at. $^{28,29}$

\section{Testing the Control Reaction}

Before testing the antioxidants, the minimal amount of $\mathrm{H}_{2} \mathrm{O}_{2}$ needed to create the most efficient reaction rate in the assay must be determined. To do this, multiple trials of the assay were run without the antioxidant with various amounts of $\mathrm{H}_{2} \mathrm{O}_{2}$ to calculate the optimal amount, so that the $\mathrm{H}_{2} \mathrm{O}_{2}$ would become a limiting reactant. If there was excess of $\mathrm{H}_{2} \mathrm{O}_{2}$, the vitamins would not work properly because there would be too much substrate, and if there was not enough $\mathrm{H}_{2} \mathrm{O}_{2}$, the reaction would not work either because the enzyme would not have a compound to reduce. For these reasons, it was crucial to correctly determine this amount. ${ }^{28,29,30}$

$1,840 \mu \mathrm{l}$ of $6.0 \mathrm{pH}$ buffer, $20 \mu \mathrm{l}$ of guaiacol, and $100 \mu \mathrm{l}$ were added into a cuvette and then placed it in a spectrophotometer. Then, various amounts of peroxide were added to determine the optimal amount that would create the most effective slope during the reaction. Each amount and concentration tested was tested in triplicates. ${ }^{28,29,30}$

When this test was finished, it was found that a $10 \mu$ of a $0.25 \% \mathrm{H}_{2} \mathrm{O}_{2}$ solution was the most efficient during the assay; therefore, this final solution was used during the next stage of testing. In order to prepare this, $4 \mathrm{~mL}$ of a $3 \% \mathrm{H}_{2} \mathrm{O}_{2}$ solution was instead mixed with $44 \mathrm{~mL}$ of water in a $45 \mathrm{~mL}$ centrifuge tube.

\section{Antioxidant Assay}

To determine whether any antioxidant or form of it that was tested was significant in mitigating the damage of $\mathrm{H}_{2} \mathrm{O}_{2}$, the previous assay was used with slight modifications. Again, these experimental protocols were adapted from previous assays to fit the purpose of this experiment. ${ }^{28,29,30} 1,870 \mu \mathrm{l}$ of $\mathrm{pH} 6.0$ buffer was added, rather than $1,840 \mu 1$ so the components added to an even 2,000 $\mu$. The first control test was run with the modified $\mathrm{H}_{2} \mathrm{O}_{2}$. This assay was run three times in order to create a baseline to compare the antioxidants to later. Then, $1,870 \mu \mathrm{l}$ of $\mathrm{pH} 6.0 \mathrm{buffer}, 10 \mu \mathrm{l}$ of $0.25 \%$ $\mathrm{H}_{2} \mathrm{O}_{2}$ and $20 \mu \mathrm{l}$ of guaiacol was placed in a cuvette. Next, the antioxidant solution was added. The amount and time of incubation of the antioxidant in the solution was determined empirically, as there was no other basis for which to determine either. For this study, $10 \mu \mathrm{l}$ of an antioxidant solution was added, and the solution incubated for 10 minutes. Then, the cuvette was placed in the spectrophotometer and the guaiacol and peroxidase were quickly added. Each reaction was run for 100 seconds. This process was repeated three times for each antioxidant that I tested. Due to limited time, these tests were performed over multiple days. The control test was re-run each day, and then used to compare to the antioxidants tested the same day. ${ }^{28}$ 


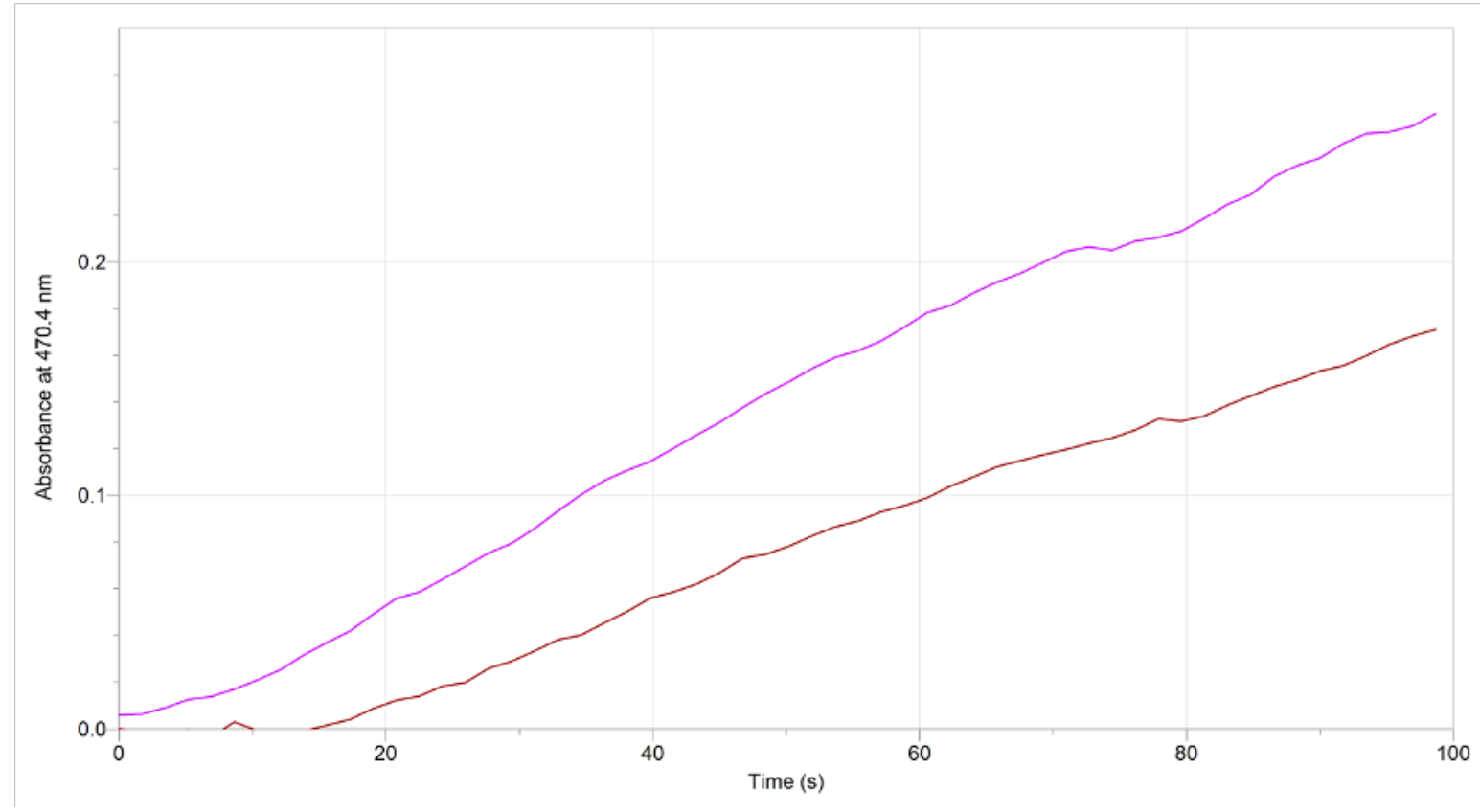

Figure 1. Sample Effect of Antioxidant on Control

Figure 1 represents a sample graph obtained from Logger Pro Software. The purple line (top) represents the control test and the red (bottom) represents an antioxidant. The slopes of these graphs measure the accumulation of oxidized guaiacol over time during the reactions.

\section{Safety and Disposal Procedures}

General laboratory safety procedures were followed; however, since the chemicals and substances being used were environmentally friendly and safe for humans, they were disposed of through a standard laboratory drain. ${ }^{30,31,32,33,34,35}$

\section{Statistical Analysis}

Slopes were calculated from data collected from 30 to 60 seconds. After collecting the slopes of the absorbances for each antioxidant, statistical analysis was conducted in order to determine which, if any, of the tested variables were significant in comparison to the control test. The slopes were calculated in $\Delta \mathrm{Abs} / \mathrm{min}$. Results were expressed as means \pm standard deviations for each treatment of the independent variable. An unpaired t-test was run to compare the means of the sample and determine if any were statistically significant. An ANCOVA test was used to evaluate if the difference in control values across the various testing days and determine if it was a significant factor in whether or not the antioxidants were statistically significant. An ANCOVA test was used to exclude the effect of the varying control values on the significance of the antioxidants. Data was considered statistically significant where $\mathrm{P} \leq 0.05 .^{28}$ The unpaired t-tests were run using Excel software. The ANCOVA test was run using JASP software. 


\section{Results}

Table 3. Antioxidant Treated Reaction Rates*

\begin{tabular}{|l|l|l|l|l|l|}
\hline \multirow{2}{*}{ Day 1 } & Control & Vitamin E (1) & Vitamin A (1) & \multicolumn{2}{l|}{ Rose Wine } \\
\cline { 2 - 6 } & $0.187 \pm 0.0057$ & $0.153 \pm 0.0488$ & $0.153 \pm 0.0266$ & $0.085 \pm 0.0095$ \\
\hline \multirow{3}{*}{ Day 2 } & Control & White Wine & Red Wine & Cranberry (RW) & $\begin{array}{l}\text { Pomegranate } \\
(\mathrm{RW})\end{array}$ \\
\cline { 2 - 6 } & $0.168 \pm 0.0080$ & $0.138 \pm 0.500$ & $0.083 \pm 0.0660$ & $0.126 \pm 0.0083$ & $0.028 \pm 0.0027$ \\
\hline \multirow{2}{*}{ Day 3 } & Control & Concord (RW) & Bai Pom & Cranberry Kroger & Bai Tea \\
\cline { 2 - 6 } & $0.130 \pm 0.0089$ & $0.014 \pm 0.0086$ & $0.067 \pm 0.0114$ & $0.009 \pm 0.0068$ & $0.071 \pm 0.0343$ \\
\hline \multirow{2}{*}{ Day 4 } & Control & Vitamin A (2) & Vitamin E (2) & Vitamin C & \\
\cline { 2 - 6 } & $0.133 \pm 0.0050$ & $0.122 \pm 0.0084$ & $0.144 \pm 0.0168$ & $0.164 \pm 0.0123$ & \\
\hline
\end{tabular}

* This table shows the absorbance per minute of each antioxidant listed directly below the name, expressed as the mean of the slopes \pm standard deviation.

Table 4. Statistical Analysis of Reaction Rates Between Control and Antioxidant Treated Reactions*

\begin{tabular}{|c|c|c|c|c|c|}
\hline \multirow{3}{*}{ Day 1} & & Vitamin E (1) & Vitamin A (1) & \multicolumn{2}{|l|}{ Rose Wine } \\
\hline & p-value ${ }^{1}$ & 0.34621 & 0.14999 & \multicolumn{2}{|l|}{0.00031} \\
\hline & t-statistic & 1.21 & 2.18 & \multicolumn{2}{|l|}{16.00} \\
\hline \multirow{3}{*}{ Day 2} & & White Wine & Red Wine & Cranberry (RW) & $\begin{array}{l}\text { Pomegranate } \\
(\mathrm{RW})\end{array}$ \\
\hline & $\mathrm{p}$-value & 0.40758 & 0.15378 & 0.00320 & 0.00037 \\
\hline & t-statistic & 1.03 & 2.21 & 6.33 & 28.73 \\
\hline \multirow{3}{*}{ Day 3} & & Concord (RW) & Bai Pom & $\begin{array}{l}\text { Cranberry } \\
\text { Kroger }\end{array}$ & Bai Tea \\
\hline & p-value & 0.00008 & 0.00202 & 0.00008 & 0.8814 \\
\hline & t-statistic & 16.31 & 7.59 & 18.61 & 2.89 \\
\hline \multirow{3}{*}{ Day 4} & & Vitamin A (2) & Vitamin E (2) & \multicolumn{2}{|l|}{ Vitamin $\mathrm{C}$} \\
\hline & p-value & 0.16073 & 0.37258 & \multicolumn{2}{|l|}{0.03245} \\
\hline & t-statistic & 1.81 & -1.10 & \multicolumn{2}{|l|}{-4.17} \\
\hline
\end{tabular}

${ }^{1}$ All $p$-values in boldface are significant at better than the .05 level for a two-tailed test.

* This shows the p-values for each antioxidant. Directly under the p-value, is the t-statistic for each antioxidant. Values were calculated using Excel software. $\mathrm{p} \leq 0.05, \mathrm{df}=2$. 
2a) Rate of Reaction for Various Antioxidants When Introduced to Hydrogen Peroxide on Day 1 (Mean \pm Standard Deviation)

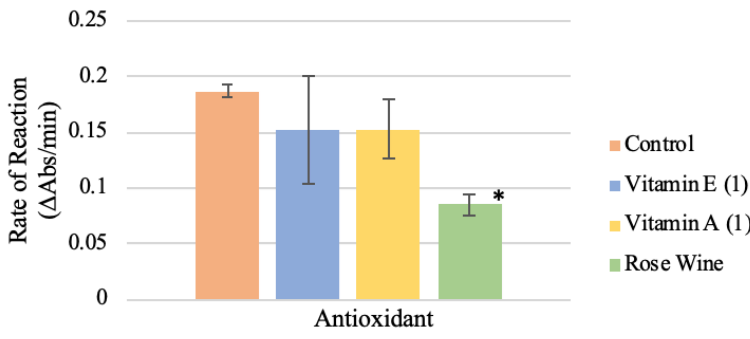

* Statistically Significant Difference at $\mathrm{P} \leq 0.05$

2c) Rate of Reaction for Various Antioxidants When Introduced to Hydrogen Peroxide on Day 3 (Mean \pm Standard Deviation)

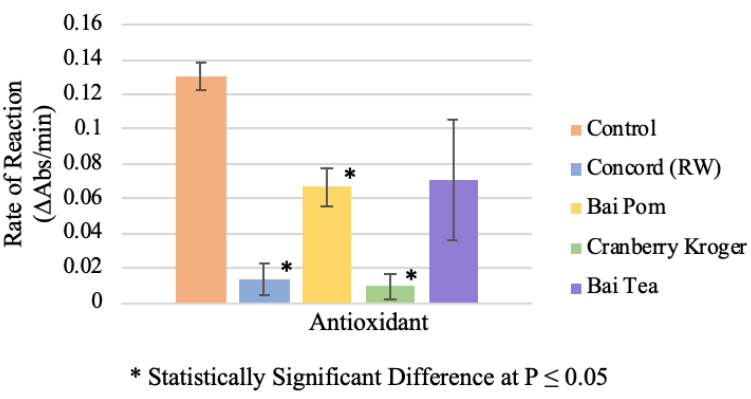

2b) Rate of Reaction for Various Antioxidants When Introduced to Hydrogen Peroxide on Day 2 (Mean \pm Standard Deviation)

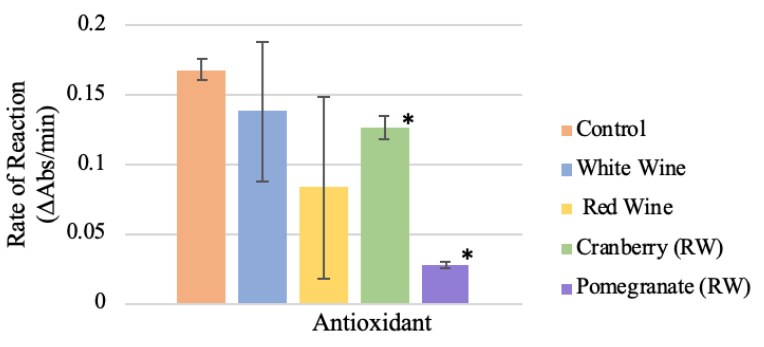

* Statistically Significant Difference at $\mathrm{P} \leq 0.05$

2d) Rate of Reaction for Various Antioxidants When Introduced to Hydrogen Peroxide on Day 4 (Mean \pm Standard Deviation)

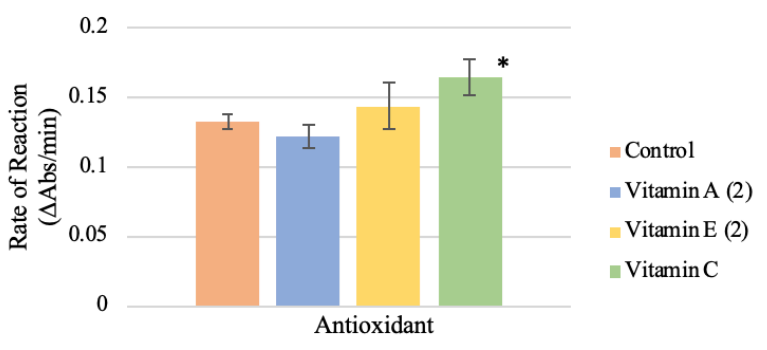

* Statistically Significant Difference at $\mathrm{P} \leq 0.05$

Figure 2. Rate of Reaction for Various Antioxidants When Introduced to Hydrogen Peroxide

Table 5. One-Way ANCOVA Results*

\begin{tabular}{|l|l|l|l|l|l|}
\hline Cases & Sum of Squares & df & Mean Square & F & p \\
\hline Baseline & $3.353 \mathrm{e}-4$ & 1 & $3.353 \mathrm{e}-4$ & 0.381 & 0.542 \\
\hline Value & 0.103 & 13 & 0.008 & 8.997 & $<0.001$ \\
\hline Residual & 0.024 & 27 & $8.791 \mathrm{e}-4$ & & \\
\hline
\end{tabular}

* As a result of this ANCOVA test, evidence shows that the baseline (control) was not a significant factor in this process, as its p-value, which was $0.542 \leq 0.05$. Therefore, the differing baseline (control) values over the four different days should not bias or limit the accuracy of the calculated t-test values.

\section{T-tests for Antioxidants Tested on Day One}

An unpaired t-test was conducted to determine if Vitamin $\mathrm{E}$ (1) [M=0.153 \pm 0.0488$]$, was significant compared to the control values for day one $[\mathrm{M}=0.187 \pm 0.0057]$. No significance was noted between the two groups, $\mathrm{t}(2)=1.21$, $\mathrm{p}=0.34621$. An unpaired t-test was conducted to determine if Vitamin A (1) $[\mathrm{M}=0.153 \pm 0.0266]$, was significant compared to the control values for day one $[\mathrm{M}=0.187 \pm 0.0057]$. No significance was noted between the two groups, $\mathrm{t}(2)=2.18, \mathrm{p}=0.14999$. An unpaired t-test was conducted to determine if Rose Wine $[\mathrm{M}=0.085 \pm 0.0095]$, was significant compared to the control values for day one $[\mathrm{M}=0.187 \pm 0.0057]$. A significant difference was noted between Rose Wine and the control $\mathrm{t}(2)=16.00, \mathrm{p}=0.00031$.

\section{T-tests for Antioxidants Tested on Day Two}

An unpaired t-test was conducted to determine if White Wine $[\mathrm{M}=0.138 \pm 0.0500]$, was significant compared to the control values for day two $[\mathrm{M}=0.168 \pm 0.0080]$. No significance was noted between the two groups, $\mathrm{t}(2)=1.03, \mathrm{p}$ 
$=0.40758$. An unpaired t-test was conducted to determine if Red Wine $[\mathrm{M}=0.083 \pm 0.0660]$, was significant compared to the control values for day two $[\mathrm{M}=0.168 \pm 0.0080]$. No significance was noted between the two groups, $\mathrm{t}(2)=12.21, \mathrm{p}=0.15378$. An unpaired $\mathrm{t}$-test was conducted to determine if Cranberry (RW) $[\mathrm{M}=0.126 \pm 0.0083]$, was significant compared to the control values for day two $[M=0.168 \pm 0.0080]$. A significant difference was noted between the two groups, $\mathrm{t}(2)=6.33, \mathrm{p}=0.00320$. An unpaired t-test was conducted to determine if Pomegranate (RW) $[\mathrm{M}=0.028 \pm 0.0027]$, was significant compared to the control values for day two $[\mathrm{M}=0.168 \pm 0.0080]$. A significant difference was noted between the two groups, $\mathrm{t}(2)=28.73, \mathrm{p}=0.00037$.

\section{T-tests for Antioxidants Tested on Day Three}

An unpaired t-test was conducted to determine if Concord (RW) $[\mathrm{M}=0.014 \pm 0.0086]$, was significant compared to the control values for day three $[\mathrm{M}=0.130 \pm 0.0089]$. A significant difference was noted between the two groups, $t(2)=16.32, p=0.00008$. An unpaired t-test was conducted to determine if Bai Pom $[\mathrm{M}=0.067 \pm 0.0114]$, was significant compared to the control values for day three $[M=0.130 \pm 0.0089]$. A significant difference was noted between the two groups, $t(2)=7.59, \mathrm{p}=0.00202$. An unpaired $\mathrm{t}$-test was conducted to determine if Cranberry Kroger $[\mathrm{M}=0.009 \pm 0.0068]$, was significant compared to the control values for day three $[\mathrm{M}=0.130 \pm 0.0089]$. A significant difference was noted between the two groups, $\mathrm{t}(2)=18.61, \mathrm{p}=0.00008$. An unpaired $\mathrm{t}$-test was conducted to determine if Bai Tea $[\mathrm{M}=0.071 \pm 0.0343]$, was significant compared to the control values for day three $[\mathrm{M}=0.130 \pm 0.0089]$. No significance was noted between the two groups, $\mathrm{t}(2)=2.89, \mathrm{p}=0.08814$.

\section{T-tests for Antioxidants Tested on Day Four}

An unpaired t-test was conducted to determine if Vitamin A (2) [M=0.122 \pm 0.0084$]$, was significant compared to the control values for day four $[\mathrm{M}=0.133 \pm 0.0050]$. No significance was noted between the two groups, $\mathrm{t}(2)=1.81$, $\mathrm{p}=0.16073$. An unpaired $t$-test was conducted to determine if Vitamin $\mathrm{E}(2)[\mathrm{M}=0.144 \pm 0.0168]$, was significant compared to the control values for day four $[\mathrm{M}=0.133 \pm 0.0050]$. No significance was noted between the two groups, $t(2)=-1.10, p=0.37258$. An unpaired $t$-test was conducted to determine if Vitamin $C[M=0.164 \pm 0.0123]$, was significant compared to the control values for day four $[\mathrm{M}=0.133 \pm 0.0050]$. A significant difference was noted between the two groups, $\mathrm{t}(2)=-4.17, \mathrm{p}=0.03245$.

\section{ANCOVA Test}

Based on my research question, the goal was to answer: How do various antioxidants comparatively help in inactivating hydrogen peroxide and limiting its negative effects? A one-way analysis of covariance was conducted using values corresponding to the antioxidants I tested as the independent variable ( 1 for all trials of Vitamin E (1), 2 for all trials of Vitamin A (1), etc.), the slope of each trial of each antioxidant as the dependent variable, and the corresponding control values for each day as the covariate. A significant relationship was not found between the dependent variable and the covariate: $F(1,27)=0.381, p=0.542$. See Table 5 .

\section{Discussion}

The goal of this research was to conduct a study that provides a more comprehensive understanding behind the chemistry of these antioxidant treated reactions in order to understand if they could be beneficial in protecting against the harmful effects of $\mathrm{H}_{2} \mathrm{O}_{2}$. As seen in the previous figures and charts, the majority of antioxidants tested showed a beneficial trend in reducing the activity of the peroxidase enzyme. The Rose Wine, all three RW juices, Bai Pom, and Cranberry Kroger all showed statistically significant effects in lowering the control. Compared to the other antioxidants, Cranberry Kroger had the lowest p-value at 0.000079, followed by Concord (RW) at 0.000084. On Day 4, two of the antioxidants that were tested seemed to increase the activity of the enzyme, therefore, increasing the average absorbance. In fact, Vitamin C was statistically significantly higher than the control for that day. Vitamin E (2) showed 
a negative trend, increasing the rate of the reaction; however, that increased rate was not statistically significant. When looking at the juices compared to the vitamins, overall the juices seemed to show a more beneficial trend in lowering the $\Delta \mathrm{Abs} / \mathrm{min}$ of the reaction.

A large portion of the data that was collected showed the positive antioxidant effects of the various juices and vitamins tested. Vitamin E (2) and Vitamin C did not support the initial hypothesis as they seemed to increase the rate of the reaction. Based on initial research, these three vitamins were commonly known for their antioxidant effects, specifically on $\mathrm{H}_{2} \mathrm{O}_{2}$. It is possible that the vitamins could have acted like an additional catalyst for the reaction; however, more research would need to be conducted in order to understand this. Although my research did not support this idea, it could have likely occurred due to the concentration of the vitamin tested, the amount added into the cuvette $(10 \mu \mathrm{l})$, or the amount of time for incubation. Because of the type of spectrophotometer that I used, it is also possible that the machine was not sensitive enough to note the minimal changes that could have been occurring in the reaction. Additionally, the concentrations of Vitamin E (1) and Vitamin A (1) created a cloudy solution which affected the absorbance levels of the spectrophotometer. Overall, the commercial items seemed to have a more positive effect overall than any of the actual vitamins that were tested.

While the antioxidant properties of the vitamins were not confirmed, the resveratrol in the Rose Wine did significantly lower the rate of the peroxidase reaction. Because all three wines did not have the same effect as the Rose Wine, it can be safely assumed that the different types of grapes and compounds used in each wine hold different antioxidant properties than each other. Red grapes have higher resveratrol levels, and because Rose Wine is commonly made with the grape skins, it is possible that the included antioxidants from the skin of the grape could have led to the reduction in the reaction. More research should be done comparing the effects of different types of grapes in response to this peroxidase-catalyzed reaction.

The absorbance measured for Cranberry Kroger was very low, and there is evidence to show that it could be helpful in protecting against ROS, in this case, $\mathrm{H}_{2} \mathrm{O}_{2}$. For this antioxidant, the slope and absorbance were extremely low until 60 seconds, where it began to increase. Although the results do not show this, as the data was collected from 30 to 60 seconds, this could likely suggest that there was not enough antioxidant introduced into the reaction, to permanently prevent the substrate from binding to the active site of the enzyme. In my initial research, I also found that some scientists had concluded that cranberries had the lowest inhibition of $\mathrm{H}_{2} \mathrm{O}_{2}$ activity. My results contradicted this because Cranberry Kroger and Cranberry (RW) were both statistically significant in lowering the rate of the reaction.

Pomegranate (RW) was statistically significant and appeared to inhibit the reaction for the entirety of the 100 seconds. My results support the previously studied notion that commercial pomegranate juice has a very high antioxidant activity compared to other juices and types of antioxidants. All three R.W. Knudsen commercial juices that were tested showed statistically significant effects inhibiting the rate of the reaction. This may suggest that an ingredient used in this company's juice products may potentially have an extremely high antioxidant activity that could be beneficial when introduced into the human diet. More research would need to be conducted centered around the components of these juices in order to make this determination.

Although Bai products are often advertised because of their antioxidant infusions, only one of the two products tested from this company showed statistically significant effects. Bai Tea showed a trend in reducing the rate of the reaction; however, there was too much variability for this antioxidant to confirm these effects. Conversely, Bai Pom significantly lowered the rate of the reaction and the results had very little variability. Because the other pomegranate juice tested (Pomegranate (RW)) showed statistically significant effects, it would be reasonable to assume that the antioxidant activity of pomegranates could be extremely important in inhibiting the damage caused by $\mathrm{H}_{2} \mathrm{O}_{2}$. More forms of pomegranates and/or pomegranate juice should be tested in order to make this determination.

Theoretically, it would have been best to test these antioxidants over the period of one day. Due to time and resources available, however, that was not possible. The ANCOVA test that was run shows that the control was not significant in affecting these results. This is positive because the slightly varying values over the four days of testing do not take away from the possible implications of these results. Similarly, due to limited resources, only three trials 
were run for each antioxidant. With more time, each antioxidant would have been tested in more trials with varying amounts and incubation periods. Due to this limitation, the results obtained in this study are not extremely generalizable. This process should be repeated more times in order to ensure validity. Nevertheless, for the amounts and incubation periods tested, many of the results showed beneficial trends in inhibiting the rate of the reaction with little variability. These results address a small gap in current science and chemical understandings and should be considered when further researching how antioxidant supplements could possibly play a role in reducing some of the harmful effects of $\mathrm{H}_{2} \mathrm{O}_{2}$.

\section{Future Directions}

This study produced many potential future directions. To begin, further research should be conducted determining the most effective amount or concentration and incubation period of each antioxidant. This would provide a better understanding of the effectiveness of each antioxidant when exposed to this peroxidase assay. Additionally, another avenue of possible research would be examining different commercial items and juices. It would be useful to determine if all products from the same company have similar effects inhibiting $\mathrm{H}_{2} \mathrm{O}_{2}$ or if the effects are limited to the specific product. Within this same area, it would also be beneficial to conduct research comparing the various parts of fruits and their antioxidant activity. This potential study could determine if the seeds, the juice, the peels, or the inner portions of these fruits are more useful in mitigating the effects of $\mathrm{H}_{2} \mathrm{O}_{2}$. Also, because I used peroxidase from a plant, in this case a turnip, this research process should be repeated using peroxidase from both animals and humans to determine if there is a difference between how the antioxidants respond to the different forms of peroxidase. An in vivo study would provide better insight as to how these antioxidants would respond in the human body.

\section{Conclusion}

Protection against ROS has generated much interest for scientists over the past few years, as they have been suggested to be a potential cause of many human diseases. Current published evidence suggests that Vitamins A, C, and E proved to have many beneficial antioxidant properties, including protecting against $\mathrm{H}_{2} \mathrm{O}_{2}$. Because of this, I believed these would be the most beneficial in reducing the slope of the reactions that they were placed into; however, they were not. The commercial items and different wines tested were overall more beneficial than the vitamins themselves. Cranberry Kroger appeared to be the most beneficial in reducing the rate of the reaction. ROS, such as $\mathrm{H}_{2} \mathrm{O}_{2}$, can be very harmful to the body, but with continued research, antioxidant supplements could prove very effective in mitigating these effects.

\section{Acknowledgments}

I would first like to thank Dr. Larry Mordan and Franklin College for the help and supplies that they provided during this research process. Additionally, I would like to thank Ms. Stephanie Davis for her guidance and encouragement.

\section{References}

1. Liu Z, Ren Z, Zhang J, et al. Role of ROS and Nutritional Antioxidants in Human Diseases. Frontiers in Physiology. 2018;9(477):1-14. doi:10.3389/fphys.2018.00477.

2. Harman D. Aging: A Theory Based on Free Radical and Radiation Chemistry. Journal of Gerontology. 1956;11(3):298-300. doi:10.1093/geronj/11.3.298. 
3. Devasagayam TP, Tilak JC, Boloor KK, Sane KS, Ghaskadbi SS, Lele RD. Free Radicals and Antioxidants in Human Health: Current Status and Future Prospects. Journal of the Association of Physicians of India. 2004;52:794-804.

4. Lane MA, Ingram DK, Roth GS. The Serious Search for an Antiaging Pill. Scientific American. 2004:3641. doi:10.1038/scientificamerican0802-36.

5. Engwa GA. Free Radicals and the Role of Plant Phytochemicals as Antioxidants Against Oxidative StressRelated Diseases. IntechOpen. 2018:49-73.

6. Kurutas EB. The importance of antioxidants which play the role in cellular response against oxidative/nitrosative stress: current state. Nutrition Journal. 2016;15(71):1-22. doi:10.1186/s12937-016-0186-5

7. McGinley C, Shafat A, Donnelly AE. Does Antioxidant Vitamin Supplementation Protect against Muscle Damage? Sports Med. 2009;39(12):1011-1032. doi:10.2165/11317890-000000000-00000.

8. Blokhina O, Virolainen E, Fagerstedt KV. Antioxidants, Oxidative Damage and Oxygen Deprivation Stress: a Review. Annals of Botany. 2003;91:179-194. doi:10.1093/aob/mcf118.

9. Lu J-M, Lin PH, Yao Q, Chen C. Chemical and molecular mechanisms of antioxidants: experimental approaches and model systems. Molecular Medicine. 2010;14(4):840-860. doi:10.1111/j.15824934.2009.00897.x.

10. King MW. Vitamins: Water and Fat Soluble. The Medical Biochemistry Page. https://themedicalbiochemistrypage.org/vitamins.php. Published 2019.

11. Copetti C, Franco FW, Machado Eda R. Acute Consumption of Bordo Grape Juice and Wine Improves Serum Antioxidant Status in Healthy Individuals and Inhibits Reactive Oxygen Species Production in Human Neuron-Like Cells. Journal of Nutrition and Metabolism. 2018;2018. doi:10.1155/2018/4384012.

12. Mounting Evidence Shows Health Benefits of Grape Polyphenols. Elsevier. https://www.elsevier.com/about/press-releases/research-and-journals/mounting-evidence-shows-health-benefits-of-grapepolyphenols. Published October 28, 2008.

13. Wang SY, Jiao H. Scavenging Capacity of Berry Crops on Superoxide Radicals, Hydrogen Peroxide, Hydroxyl Radicals, and Singlet Oxygen. Journal of Agricultural and Food Chemistry. 2000;48:5677-5684. doi:10.1021/jf000766i. 
14. Gil MI, Tomás-Barberán FA, Hess-Pierce B, Holcroft DM, Kader AA. Antioxidant Activity of Pomegranate Juice and Its Relationship with Phenolic Composition and Processing. Journal of Agricultural Food and Chemistry. 2000;48:4581-4589. doi:10.1021/jf000404a.

15. O'Byrne DJ, Devaraj S, Grundy SM, Jialal I. Comparison of the antioxidant effects of Concord grape juice flavonoids and $\alpha$-tocopherol on markers of oxidative stress in healthy adults. The American Journal of Clinical Nutrition. 2002;76:1367-1374. doi:10.1093/ajcn/76.6.1367.

16. Duthie SJ, Jenkinson AME, Crozier A, et al. The effects of cranberry juice consumption on antioxidant status and biomarkers relating to heart disease and cancer in healthy human volunteers. European Journal of Nutrition. 2006;45:113-122. doi:10.1007/s00394-005-0572-9.

17. Guo S, Deng Q, Xiao J, Xie B, Sun Z. Evaluation of Antioxidant Activity and Preventing DNA Damage Effect of Pomegranate Extracts by Chemiluminescence Method. Journal of Agricultural and Food Chemistry. 2007;55:3134-3140. doi:10.1021/jf063443g.

18. Caillet S, Côté J, Doyon G, Sylvain J-F, Lacroix M. Antioxidant and antiradical properties of cranberry juice and extracts. Food Research International. 2011;44:1408-1413. doi:10.1016/j.foodres.2011.02.019.

19. Konyalioglu S, Armagan G, Yalcin A, Atalayin C, Dagci T. Effects of resveratrol on hydrogen peroxideinduced oxidative stress in embryonic neural stem cells. Neural Regeneration Research. 2013;8(6):485495. doi:10.3969/j.issn.1673-5374.2013.06.001.

20. Bosutti A, Degens H. The impact of resveratrol and hydrogen peroxide on muscle cell plasticity shows a dose-dependent interaction. Scientific Reports. 2015;5(8093):1-11. doi:10.1038/srep08093.

21. Les F, Prieto JM, Arbonés-Mainar JM, Valero MS, López V. Bioactive properties of commercialised pomegranate (Punica granatum) juice: antioxidant, antiproliferative and enzyme inhibiting activities. Food and Function. 2015;6(6):2049-2057. doi:10.1039/c5fo00426h.

22. Cásedas G, González-Burgos E, Smith C, López V, Gómez-Serranillos MP. Regulation of redox status in neuronal SH-SY5Y cells by blueberry (Vaccinium myrtillus L.) juice, cranberry (Vaccinium macrocarpon A.) juice and cyanidin. Food and Chemical Toxicology. 2018;118:572-580. doi:10.1016/j.fct.2018.05.066.

23. Bjelakovic G, Nikolova D, Gluud LL, Simonetti RG, Gluud C. Mortality in Randomized Trials of Antioxidant Supplements for Primary and Secondary Prevention. Journal of the American Medical Association. 2007;297(8):842-857. doi:10.1001/jama.297.8.842.

24. NIH State-of-the-Science Panel. National Institutes of Health State-of-the-Science Conference Statement: Multivitamin/Mineral Supplements and Chronic Disease Prevention. Annals of Internal Medicine. 2006;145(5):364-371. doi:10.1093/ajen/85.1.257S. 
25. Huang H-Y, Caballero B, Chang S, et al. The Efficacy and Safety of Multivitamin and Mineral Supplement Use To Prevent Cancer and Chronic Disease in Adults: A Systematic Review for a National Institutes of Health State-of-the-Science Conference. Annals of Internal Medicine. 2006;145(5):372-385.

26. Hercberg S, Galan P, Preziosi P, et al. The SU.VI.MAX Study: a randomized, placebo-controlled trial of the health effects of antioxidant vitamins and minerals. JAMA Internal Medicine. 2004;164:2335-2342. doi:10.1001/archinte.164.21.2335.

27. Young IS, Woodside JV. Antioxidants in health and disease. Journal of Clinical Pathology. 2001;54:176186. doi:10.1136/jcp.54.3.176.

28. Franklin College Cell Biology Lab Manual. 2019.

29. Enzyme Activity Guided Inquiry Lab Turnip Peroxidase. Flinn Scientific, Inc. 2018.

30. AP Biology Lab Manual. Enzyme Activity Investigation 13. https://secure-media.collegeboard.org/digitalServices/pdf/ap/bio-manual/Bio_Lab13-EnzymeActivity.pdf. 2001.

31. Safety Data Sheet Alpha-Tocopherol. Cayman Chemical Company. 2018.

32. Safety Data Sheet Guaiacol. Cayman Chemical Company. 2017.

33. Safety Data Sheet Hydrogen Peroxide 50\% Oxypure. PeroxyChem. 2015.

34. Safety Data Sheet L-Ascorbic Acid. Cayman Chemical Company. 2018.

35. Safety Data Sheet Vitamin A. Cayman Chemical Company. 2017.

36. Bartee L. Enzymes. Principles of Biology: Biology 211212 and 213. https://openoregon.pressbooks.pub/mhccmajorsbio/chapter/6-6-enzymes/. Accessed November 17, 2019.

37. The Meaning of Reduction and Oxidation. ChemTeam. https://www.chemteam.info/Redox/Meaning-ofRedox.html. Accessed November 18, 2019.

38. Differences between in vitro, in vivo, and in silico studies. Marshall Protocol Knowledge Base. https://mpkb.org/home/patients/assessing_literature/in_vitro_studies. Published September 1, 2019. 
39. NCI Dictionary of Cancer Terms. National Cancer Institute. https://www.cancer.gov/publications/dictionaries/cancer-terms/def/assay.

40. What is a cuvette and how is it used? CM Scientific. https://www.cmscientific.com/cuvette_info.php. 